\title{
Changes in Basal Metabolic Rate following Gastric Bypass surgery
}

\author{
F. Naseer, R.K. Price, A. Boyd, T. Redpath, D. Liddy and M.B.E. Livingstone \\ Nutrition Innovation Centre for food and Health (NICHE), Ulster University, School of Biomedical Science, \\ Coleraine BT52 1SA, UK
}

Gastric Bypass (GB) surgery continues to be one of the few effective treatments for sustainable weight loss in obese individuals ${ }^{(1)}$. Apart from the primary weight loss mechanisms of energy restriction and malabsorption, studies have postulated that GB is associated with an increased shift in energy expenditure (EE) that contributes to sustained weight loss in the longer-term (2-5). However this positive effect on EE in humans remain equivocal. The effect has mainly been observed in experimental animal studies which adjusted EE for changes in total body weight (TBW) and body composition (BC) observed postoperatively ${ }^{(3-5)}$.

The aim of this study was to assess the impact of GB on Basal Metabolic Rate (BMR) in 17 GB patients ( 5 males; BMI $45 \cdot 8 \pm 6 \cdot 3$ $\mathrm{kg} / \mathrm{m}^{2} ; 47 \cdot 2 \pm 13 \mathrm{y}$ ) and 13 time-matched and weight-stable controls (5 males; BMI $\left.25 \cdot 8 \pm 4 \cdot 3 \mathrm{~kg} / \mathrm{m}^{2} ; 39.4 \pm 15 \cdot 1 \mathrm{y}\right)$ at baseline (one month pre-surgery) and at three months post-surgery. BMR was measured under standardised conditions using indirect calorimetry (ECAL, Metabolic Health Solutions). Lean body mass (LBM) and fat mass (FM) were measured using dual energy X-ray absorptiometry (DEXA, GE Healthcare). BMR values were expressed per kg of TBW, LBM and FM. A paired sample $t$-test was used to compare changes in BMR between baseline and 3 months post-surgery.

Mean absolute BMR (kJ/day), TBW $(\mathrm{kg}), \mathrm{BMI}\left(\mathrm{kg} / \mathrm{m}^{2}\right)$, LBM $(\mathrm{kg})$ and FM $(\mathrm{kg})$ significantly decreased by $16 \cdot 1 \pm 21 \%, 16 \cdot 9 \pm 4 \cdot 3 \%$, $16 \cdot 7 \pm 4 \cdot 4 \%, 9 \cdot 8 \pm 4 \cdot 7 \%$ and $24 \cdot 7 \pm 7 \cdot 1 \%$ respectively in patients $(\mathrm{P}<0.05)$ and by $-0 \cdot 5 \pm 24.4 \% 1 \cdot 1 \pm 2 \cdot 4 \%, 0 \cdot 4 \pm 2 \cdot 8 \%, 0 \cdot 3 \pm 3 \cdot 1 \%$ and $2 \cdot 1 \pm 8 \cdot 1 \%$ respectively in controls (all non-significant). When expressed relative to TBW and LBM, GB attenuated the compensatory reductions in BMR while BMR expressed relative to FM increased three months post-surgery in patients.

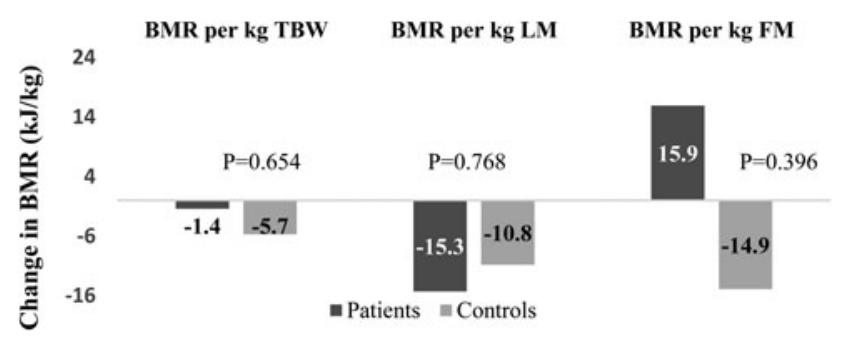

Fig. 1. Changes in BMR expressed per kg of TBW, LBM, and FM at three months post-surgery in patients and controls. $F M$ fat mass, $L M$ lean body mass, $T B W$ total body weight.

In conclusion, weight loss following GB is associated with a favourable increase in BMR when expressed relative to postoperative changes in TBW and BC. This may contribute to the successful weight loss outcomes observed postoperatively. Further research is warranted to elucidate the underlying mechanism for this apparent beneficial impact of GB on BMR.

1. Colquitt J, Pickett K, Loveman E et al. (2014) Cochrane Database of Syst Rev 8(8).

2. Faria S, Faria O, Buffington C et al. (2012) Obes Surg 22(9), 1450-1455.

3. Hao Z, Mumphrey M, Townsend R et al. (2016) Obes Surg 26(9), 2173-2182.

4. Nestoridi E, Kvas S, Kucharczyk J et al. (2012) Endocrinology 153(5), $2234-44$.

5. Stylopoulos N, Hoppin AG \& Kaplan LM (2009) Obesity 17(10), 1893-47. 